\title{
La Percepción de los Agricultores sobre el Problema del Tizón Tardío o Rancha (Phytophthora infestans) su Manejo: Estudio de Casos en Cajamarca, Perú
}

\author{
Oscar Ortiz ${ }^{1}$, Paul Winters ${ }^{2}$, Hugo Fano ${ }^{3}$
}

Los agricultores que cultivan papa en zonas tropicales montañosas enfrentan varios problemas que limitan la productividad y producción. Sin embargo, poco se conoce sobre los efectos del tizón tardío, el conocimiento de los agricultores y el manejo de esta enfermedad a nivel de campo en países en desarrollo. Este estudio se llevó a cabo en Cajamarca, Perú, durante la campaña agrícola de 1997-1998. Un total de 131 agricultores participaron en entrevistas formales e informales y brindaron sus campos para evaluaciones.

Los agricultores entrevistados sembraban alrededor de 0.6 ha con papa, mayormente bajo condiciones de secano. Ellos indicaron que el tizón tardío era el principal problema en la zona, el cual reducía la producción significativamente. En general, la mayoría de entrevistados identificaron claramente las condiciones climáticas que favorecen la enfermedad; pero no conocían que esta enfermedad es causada por un microorganismo y tampoco tenían la habilidad para diagnosticarla correctamente.

La mayoría de agricultores identificaron al control químico como la principal práctica de control y usaron 6.6 aplicaciones en promedio. El uso de variedades resistentes y diferentes fechas de siembra también fueron mencionados, pero en menor medida. Los fungicidas más usados en la zona fueron el mancozeb y el metalaxyl. La evidencia indicó que el uso de fungicidas está significativamente asociado con la edad de los agricultores, el nivel de educación, el tamaño de la finca, la fecha de siembra y

1 Ing. Agr., M.Sc., Ph.D. Especialista en Extensión Agrícola. Departamento de Ciencias Sociales. Centro Internacional de la Papa (CIP). Apartado 1558, Lima 12, Perú.

${ }^{2}$ Ph.D. Economista. University of New England, Armidale, NSW 2351, Australia.

3 Economista. Departamento de Ciencias Sociales. Centro Internacional de la Papa (CIP). Apartado 1558, Lima 12, Perú. 
la ubicación de la finca; pero no está correlacionado con el uso de variedades.

Las evaluaciones de campo demostraron que la severidad de ataque por tizón tardío está significativa e indirectamente correlacionada con el rendimiento del cultivo de papa. Se estableció que $20 \%$ de incremento en severidad tiende a reducir aproximadamente 1 t/ha de rendimiento. La fecha de siembra, la fecha de evaluación, el número de aplicaciones de fungicidas y las variedades de papa sembradas estuvieron correlacionados significativamente con la severidad de la enfermedad.

La evidencia del estudio indica que es necesario investigar y difundir métodos de control eficientes contra el tizón tardío y que estos métodos serían rentables, ya que esta enfermedad es el principal factor que disminuye el rendimiento del cultivo de la papa según la percepción de los agricultores y los datos provenientes de evaluaciones de campos.

Palabras claves adicionales: tizón tardío, diagnóstico socioeconómico, conocimiento campesino, percepción de agricultores.

Aceptado para publicación: noviembre 1998.

\section{Farmers' Perception of Late Blight (Phytophthora Infestans) and its Management: Case Studies in Cajamarca, Peru}

\section{Summary}

Farmers who grow potatoes in mountainous areas face a number of problems that constrain production and productivity. However, little is know about the effects of diseases such as late blight, farmers' knowledge and management of it at field level in developing countries. This paper presents the results of a base line study that describes farmers' knowledge and practices to control this disease. The study was carried out in Cajamarca, Peru during the 1997-1998 cropping season. A total of 131 farmers participated in both formal and informal interviews and field evaluations. 
Farmers participating planted about 0.6 ha with potatoes, mainly under rain fed conditions. They perceived that late blight was the most important problem, which reduces significantly potato productivity. In general, most farmers clearly identified the climatic conditions that favour late blight development; but they do not know that this disease is caused by a microorganism, and they tend to misdiagnose the disease.

The majority of farmers consider the use of fungicides as the main control practice and they used an average of 6.6 sprays in the cropping season. The use of resistant varieties and planting dates were also mentioned to a lesser extent. Mancozeb and metalaxyl were the most commonly used fungicides in the area. Evidence showed that fungicide use is associated significantly with farmers' age and education level, and also with farm size, planting date and location, but not with varietals use.

Field evaluation showed that late blight severity is significantly but negatively correlated with potato yields. It was estimated that $20 \%$ increase in late blight severity tends to reduce yield by $1 \mathrm{t} / \mathrm{ha}$. Planting date, date of evaluation, number of fungicide sprays and use of varieties were correlated to a significant extent with late blight severity.

Evidence from this study suggest that there is the need to implement research and extension projects related to late blight control, and that these activities would be profitable because this disease is the main constrain that reduces potato yields according to both farmers' perceptions and field evaluations.

Additional index words:

late blight, socioeconomic studies, indigenous knowledge, farmers' perceptions.

\section{Introducción}

Los agricultores que cultivan papa en zonas tropicales montañosas enfrentan una serie de problemas de tipo biótico y abiótico, los cuales limitan la productividad y producción. Entre los factores bióticos más importantes se encuentra el tizón tardío o rancha producido por el hongo Phytophthora infestans. En realidad los agricultores peruanos 
han enfrentado esta enfermedad desde hace por lo menos un siglo. Torres (1896) describe una enfermedad que destruía el follaje de la papa en los valles aledaños a Lima, la cual presumiblemente era el tizón. Esta enfermedad se ha agudizado en los últimos años y hay evidencia de que está causando mayores daños a los cultivos de papa (Pérez, W. comunicación personal, 1988).

Este artículo tiene como objetivo el presentar los resultados de un diagnóstico ${ }^{4}$ que describe el conocimiento y la forma en que los agricultores de la zona de Cajamarca, Perú enfrentan este problema: conocer los factores que influyen en el ataque del tizón tardío será útil para mejorar la investigación participativa para el manejo integrado de esta enfermedad.

\section{El proceso de investigación}

\section{La selección del lugary de los informantes:}

El estudio se concentró en las provincias de Cajamarca, San Miguel y Contumazá en el Departamento de Cajamarca, Perú por dos razones importantes. Primero, porque es una zona donde la mayoría de los agricultores son de subsistencia y, segundo, porque en Cajamarca, específicamente en la Provincia de San Miguel, se está desarrollando la experiencia piloto de implementación de escuelas de campo (van de Fliert, 1993; Nelson et al 1998), el cual es un proyecto colaborativo entre el CIP y CARE-Perú. El objetivo de las escuelas de campo es desarrollar el manejo integrado del tizón tardío con participación de los agricultores.

El proceso de selección de los lugares se basó en un muestreo dirigido. Se seleccionaron las provincias de acuerdo al riesgo de ataque de tizón tardío. De esta manera se seleccionó a la provincia de Cajamarca como de mediano riesgo y las provincias de Contumazá y San Miguel como de alto riesgo. En estas provincias se seleccionaron distritos específicos de acuerdo a la importancia de la papa. En total se tuvo acceso a información de 131 agricultores.

\section{El método para colectar información durante el estudio:}

Se usaron varias técnicas para colectar información de los agricultores, de tal manera de comparar y complementar los datos de varias fuentes

\footnotetext{
${ }^{4}$ Este estudio fue financiado por el Fondo para el Desarrollo Internacional de los Países Exportadores de Petróleo (OPEC) y realizado gracias a la colaboración de CARE-Perú.
} 
y con diferentes técnicas, lo cual es llamado triangulación por Krueger (1994). La principal técnica para colectar información fue el uso de un cuestionario con preguntas semi-estructuradas. Otra forma de colección de datos con un enfoque más cualitativo fueron los grupos de discusión o grupos focales (Morgan y Krueger, 1993) con los agricultores para discutir sobre el manejo del tizón tardío. Las discusiones grupales fueron útiles para generar más información, complementar y enriquecer la generada a través de los cuestionarios. También se colectó información utilizando el método de la observación no participante sugerido por Röling (1990). La evaluación directa de las parcelas de papa fue otra forma de colección de datos. Las parcelas fueron evaluadas básicamente en dos aspectos: el daño por tizón tardío y el rendimiento.

\section{Las características de los informantes y las fincas}

\section{Los informantes:}

En términos generales, los agricultores informantes eran de subsistencia; es decir aquellos que siembran básicamente para satisfacer sus necesidades de autoconsumo y venden sólo los excedentes (Ortiz et al 1996). Sin embargo, en la Provincia de Contumazá los agricultores tenían vínculos comerciales con el mercado de la costa.

Respecto a las actividades que generan ingresos para los agricultores, la evidencia demostró que todos los agricultores se dedicaban a la agricultura y $73 \%$ de ellos a la ganadería como principales fuentes de ingreso. Menos de $30 \%$ de agricultores realizaban actividades complementarias como trabajo asalariado temporal, comercio y artesanía.

El promedio del tamaño total de las fincas fue de 8.7 ha, lo cual es relativamente alto para la zona estudiada, en la cual según INEI (1996) la mayoría de las fincas tienen menos de 5 ha. Las fincas son básicamente de secano. Esta característica obliga a los agricultores a sembrar la mayor parte de sus tierras durante la época de lluvia, que es a su vez la época de mayor ataque del tizón tardío o rancha de la papa.

El área cultivada, sin embargo, se ajusta a los promedios normales de la zona. Los agricultores cultivaban un promedio de 1.9 ha divididas entre 3 a 5 parcelas con diversos cultivos. En total se identificaron 
hasta 15 cultivos principales. En la Tabla 1 se indican las áreas promedio dedicadas a tos principales cultivos en la zona en estudio. En primer lugar se aprecia que la papa es un cultivo importante en las tres provincias con un área promedio de 0.63 ha; sin embargo, en el caso de Cajamarca, los cereales (trigo, cebada, avena y centeno) son más importantes en términos de área. Lo mismo sucede con las legumbres (arvejas) en la provincia de San Miguel. Además, los agricultores manejaban diversos tipos de ganado, tales como vacuno, ovino, equino y animales menores. Esta diversidad de actividades agropecuarias, que se suma a las actividades externas a la finca, también ha sido observada en Cajamarca por Gálvez et al (1988), Sánchez et al (1993) y Ortiz y Valdez (1993),

Tabla 1. Los cultivos más importantes en la zona estudiada ( $N=131)$.

\begin{tabular}{|l|c|c|c|c|}
\hline Área sembrada (ha) & Cajamarca & Contumazá & San Miguel & Promedio \\
\hline Papa & 0.58 & 0.79 & 0.59 & 0.63 \\
Maíz & 0.10 & 0.03 & 0.25 & 0.14 \\
Cereales $^{\perp}$ & 1.20 & 0.10 & 0.16 & 0.57 \\
Legumbres $^{<}$ & 0.13 & 0.13 & 0.67 & 0.32 \\
Tubérculos andinos $^{3}$ & 0.08 & 0.00 & 0.01 & 0.04 \\
Otros $^{4}$ & 0.16 & 0.35 & 0.06 & 0,17 \\
\hline
\end{tabular}

${ }^{1}$ Incluye cebada, trigo, centeno y avena

2 Incluye haba, arveja y lupino

${ }^{3}$ Incluye oca, dluco y mashua

${ }^{4}$ Incluye quinua, kiwicha y hortalizas varias

\section{Percepción de los agricultores sobre la importancia del tizón tardío}

La mayoría de tos agricultores (67\%) que participaron en el estudio calificaron al tizón tardío como el problema más importante y $24 \%$ lo calificaron como el segundo problema en orden de importancia. Sólo 9\% de los informantes lo calificaron en tercero o cuarto lugar. Los agricultores también mencionaron otros problemas, tal es el caso del gorgojo de los andes (Premmnotrypes vorax) que para $10 \%$ de informantes era el problema principal y para $61 \%$ era el segundo 0 tercer problema en orden de importancia. Algunos agricultores (20\%) también identificaron a las heladas como una plaga, lo cual sugiere que ellos confunden las verdaderas causas de las plagas de los cultivos. 
Hubieron también otras plagas que fueron menos importantes como la pulga saltona o Epitrix, la pudrición de tubérculos, la polilla de la papa, insectos de hoja como Diabrotica, otros hongos como Alternaria y otras plagas menores como la gallina ciega (Phyllophaga sp). Ortiz y Valdez (1993) también reportan al tizón, las heladas y el gorgojo de los Andes como los principales problemas identificados por agricultores paperos en la provincia de Cajamarca. Horton et al (1980) reportan problemas similares de insectos en los Andes Centrales del Perú.

Es posible ponderar las calificaciones dadas por los agricultores asignándole un puntaje. Por ejemplo, si le asigna 5 puntos cada vez que un agricultor califica una plaga como primer problema, 4 puntos al segundo, 3 al tercero, etc. Luego se normalizan los puntajes de tal manera que si todos los agricultores hubieran mencionado al tizón tardío como primer problema, éste tendría una ponderación de 100. La Figura 1 muestra el resultado de este cálculo en las tres provincias evaluadas. Se aprecia claramente que el tizón tardío es el principal problema en los tres lugares, con una ligera menor incidencia en Cajamarca. La importancia de los otros problemas de plagas, incluidas las heladas en la percepción de los agricultores, depende de cada lugar. Por ejemplo, el gorgojo de los Andes es más importante en Contumazá y menos importante en San Miguel. Las heladas son más importantes en Cajamarca y mucho menos en Contumazá. El Epitrix parece atacar más en San Miguel. Los otros problemas (pudriciones, polilla, otros insectos y hongos) tienen poca importancia en opinión de los agricultores.

Se preguntó a los agricultores sobre sus percepciones acerca de las pérdidas que ocasionan las enfermedades y los insectos de la papa. Las respuestas fueron bastante consistentes y fue claro que los agricultores consideran al tizón tardío como el problema que reduce más drásticamente los rendimientos. La mitad de los informantes indicaron que esta enfermedad puede reducir el rendimiento en más del $50 \%$ en el peor de los casos. $25 \%$ de ellos opinaron que el tizón tardío puede reducir el rendimiento entre $26 \%$ a $50 \%$ y el otro $25 \%$ de ellos indicaron que el tizón reducía menos del 25\% del rendimiento. 


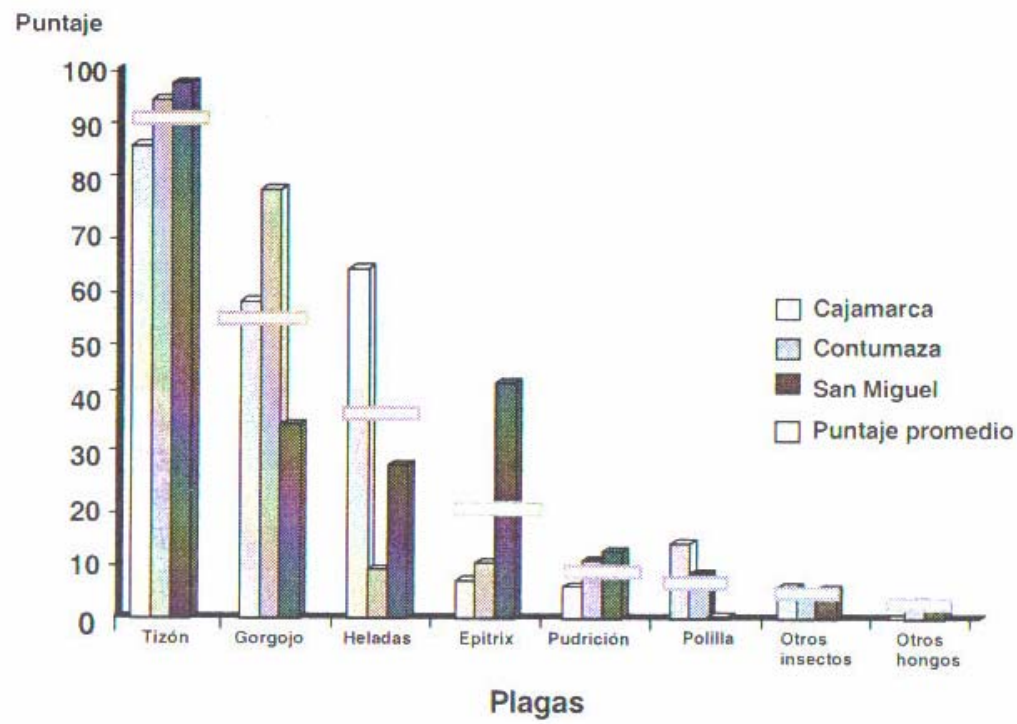

Figura 1. Problemas de plagas (incluidas las heladas) percibidas por los agricultores* Puntajes ponderados y normalizados $(N=131)$.

Según $40 \%$ de agricultores, el gorgojo de los Andes es otra plaga que reduce la calidad de la cosecha en más de $50 \%$ cuando su ataque es severo. Hubieron otras plagas mencionadas por los agricultores que según su opinión no tienen mucha importancia en términos de reducir los rendimientos. Por ejemplo, en el caso de la polilla de la papa sólo $20 \%$ de entrevistados indicaron que en el peor caso puede afectar más del $50 \%$ de la producción o del volumen almacenado. Menos de $15 \%$ de agricultores indicaron que la Alternaria (llamada "rancha amarilla" por ellos) reducía más del $50 \%$ del rendimiento de la papa. El caso de la pudrición fue aun menos importante. Sólo 8\% de agricultores indicaron que este problema puede reducir más del $50 \%$ del rendimiento.

En conclusión, se puede decir que desde el punto de vista de los agricultores de la zona estudiada, el tizón tardío es el principal problema en el cultivo de la papa, no sólo por que se presenta continuamente y en todos los lugares donde se siembra papa, sino porque puede reducir drásticamente los rendimientos, especialmente en años cuando las condiciones de humedad son favorables para el 
microorganismo. Sin embargo, en cada lugar también hay otras plagas que pueden afectar seriamente la papa, especialmente el caso del gorgojo de los Andes. Este resultado, sin embargo, no se puede generalizar para todo el Perú. Por ejemplo, Horton et al (1980) reportan que el tizón no es un problema importante del cultivo de la papa en el Valle del Mantaro. Es decir, habría que hacer un estudio más detallado para determinar las zonas paperas que están bajo riesgo de ser afectadas por esta enfermedad.

\section{El conocimiento de los agricultores sobre el tizón tardío y sobre las prácticas de control que usan}

\section{Conocimiento de los agricultores sobre el origen de la enfermedad:}

Fue interesante observar que los agricultores reconocían claramente las condiciones climáticas que facilitan la aparición de la enfermedad. Casi la mitad de ellos (48\%) identificaron al exceso de lluvia, otros (38\%) identifican a la neblina como la causa y otros (18\%) identificaron a combinación de periodos de lluvia con periodos de sol como causa del tizón. Algunos de ellos (8\%) mencionaron la presencia de rayos 0 relámpagos es la causa de la enfermedad. Sólo $9 \%$ de los informantes indicaron que el tizón es causado por un hongo que es favorecido por la humedad. En realidad, la mayoría de agricultores desconoce el concepto de hongo y, por lo tanto, relacionan más a la enfermedad con las condiciones climáticas de excesiva humedad que es lo que ellos han podido observar en sus campos. Similares observaciones reporta Bentley (1990) en el caso de enfermedades fungosas del maíz en América Central.

\section{Reconocimiento de los síntomas del tizón:}

La mayoría de agricultores (88\%) identificaron como tizón o rancha a cualquier mancha foliar o quemado de hojas o tallos. Sólo un $4 \%$ de ellos identificó el síntoma claramente como manchas oscuras en el haz de las hojas con un polvillo blanquecino en el envés. Respecto a los lugares de la planta donde ellos han observado los síntomas, 74\% de informantes indicaron que los síntomas se presentan en las hojas y los tallos. Otros (26\%) indican que además de hojas y tallos, también hay síntomas en los tubérculos, los cuales presentan manchas marrones en la pulpa, pudriciones o endurecimiento.

Por otro lado, los agricultores identificaron dos tipos de tizón tardío o rancha. El primero es llamada "rancha negra" que corresponde al causado por el Phytophthora infestans que se presenta mayormente durante la época lluviosa (entre noviembre a abril). El segundo es 
llamado "rancha amarilla" y corresponde a enfermedades foliares que se presentan mayormente en las siembras tempranas entre junio a noviembre y son causadas por hongos como Alternaria o Phoma.

La evidencia sugiere que la mayoría de agricultores carecen del conocimiento necesario para identificar apropiadamente los síntomas del tizón tardío, los cuales son frecuentemente confundidos con otras enfermedades foliares. Esta confusión podría estar influyendo la decisión de aplicar fungicidas cuando no es necesario hacerlo.

\section{Conocimiento de los agricultores sobre las prácticas de control:}

Para el control del tizón tardío, la mayoría de entrevistados (94\%) mencionó al uso de fungicidas como la práctica más común, aunque ellos se refirieron a los productos químicos con el término de "remedios". Estos agricultores consideraban que los productos químicos eran la única forma de controlar la enfermedad. Sin embargo, cuando se discutió el tema en los grupos, los agricultores mencionaron otras estrategias que utilizaban para tratar de disminuir los efectos del tizón en la papa. Una de estas estrategias era la de sembrar lo más temprano posible en la campaña agrícola. Es decir sembrar en lo que ellos llaman "campaña chica" que se inicia aproximadamente en junio. Si los agricultores siembran entre junio a septiembre, el riesgo de ataque del tizón se reduce considerablemente ya que existe menor humedad en ese periodo. Sin embargo, esta estrategia sólo puede ser usada en terrenos que tienen acceso a riego. El uso de variedades resistentes también fue mencionada como una estrategia para reducir la incidencia de la enfermedad durante las discusiones grupales. Sólo dos agricultores en la zona de Cajamarca, mencionaron haber ensayado extractos vegetales para controlar el tizón; pero que aún no estaban seguros de su eficiencia

\section{Las prácticas de control usadas por los agricultores}

\section{La época de siembra:}

En las tres Provincias analizadas fue claro que menos de $20 \%$ de campos de papa se siembran entre junio y septiembre (época seca), reduciendo de esta manera el riesgo de ataque del tizón. Estos campos generalmente tienen riego o están ubicados a mayor altitud y son más húmedos. La gran mayoría de agricultores siembra entre octubre a diciembre y cultiva la papa durante la época lluviosa. Esto eleva el riesgo de tener pérdidas debidas al ataque del tizón tardío. En realidad, es poco lo que los agricultores pueden hacer para variar las épocas de siembra ya que la mayoría posee terrenos en secano. 


\section{El uso de variedades con resistencia al tizón:}

Durante las entrevistas con los agricultores se trató de identificar las razones por las cuales los agricultores prefieren una variedad de papa. En promedio, $85.5 \%$ de entrevistados mencionó a la resistencia al tizón como una característica deseada en una variedad de papa. El buen rendimiento también fue mencionado por $70.2 \%$ de entrevistados como una característica preferida. Los agricultores también mencionaron la buena calidad culinaria (64.6\%), precocidad (35.9\%), buena aceptación en el mercado (35.1\%), resistencia a sequía (6.9\%) y resistencia a la pudrición (5.3\%).

Tabla 2: Razones por las cuales los agricultores prefieren las variedades más comunes en la zona.

\begin{tabular}{|l|l|l|l|l|l|l|l|}
\hline Característica preferida: & $\begin{array}{l}\text { Yun- } \\
\text { gay }\end{array}$ & $\begin{array}{l}\text { Can- } \\
\text { chan }\end{array}$ & $\begin{array}{l}\text { Liber- } \\
\text { teña }\end{array}$ & $\begin{array}{l}\text { Ama- } \\
\text { rilis }\end{array}$ & $\begin{array}{l}\text { Perri- } \\
\text { choli }\end{array}$ & $\begin{array}{l}\text { Colo- } \\
\text { rada }\end{array}$ & $\begin{array}{l}\text { Renaci- } \\
\text { miento }\end{array}$ \\
\hline Resistencia al tizón & $29.7 \%$ & $20.0 \%$ & $65.2 \%$ & $83.0 \%$ & $70.8 \%$ & $0.0 \%$ & $13.3 \%$ \\
Rendimiento & $66.2 \%$ & $22.9 \%$ & $27.3 \%$ & $45.8 \%$ & $25.0 \%$ & $25.0 \%$ & $53.3 \%$ \\
Calidad culinaria & $31.1 \%$ & $32.9 \%$ & $42.4 \%$ & $23.7 \%$ & $16.7 \%$ & $87.5 \%$ & $53.3 \%$ \\
Precocidad & $6.7 \%$ & $40.0 \%$ & $4.5 \%$ & $11.9 \%$ & $8.3 \%$ & $0.0 \%$ & $0.0 \%$ \\
Aceptación en el & $12.2 \%$ & $17.1 \%$ & $30.3 \%$ & $5.1 \%$ & $8.3 \%$ & $12.5 \%$ & $6.7 \%$ \\
$\begin{array}{l}\text { Resistencia a la } \\
\text { Resistencia a } \\
\text { humedad o pudrición }\end{array}$ & $1.4 \%$ & $4.2 \%$ & $7.5 \%$ & $1.7 \%$ & $0.0 \%$ & $0.0 \%$ & $0.0 \%$ \\
\hline
\end{tabular}

Es claro, por la evidencia presentada, que los agricultores prefieren una variedad mayormente por su resistencia al tizón, su buen rendimiento y su calidad culinaria. Sin embargo, esto depende de cada variedad. Por ejemplo en la Tabla 2 se aprecian las razones por las cuales los agricultores prefieren las variedades más comunes en la zona. Los porcentajes se refieren a la proporción efe agricultores que mencionaron cada variedad. Se tiene el caso de Yungay que es preferida por su buen rendimiento en $76.2 \%$ de casos. Canchan es preferida en $40 \%$ de los casos por su precocidad y en $31.1 \%$ de casos por su calidad culinaria. Liberteña es preferida por $65.2 \%$ de casos por su resistencia al tizón (especialmente en zonas con mediano riesgo de ataque como en la Provincia de Cajamarca). Amarilis y Perricholi son claramente preferidas por su resistencia al tizón (mencionado en $83 \%$ y $70.8 \%$ de los casos respectivamente). La variedad Colorada, que es una variedad nativa, es preferida por su calidad culinaria (87.5\%) y la variedad Renacimiento es preferida mayormente por su buen rendimiento y buena calidad culinaria. Aunque en general la característica preferida es la resistencia al tizón, algunas variedades se prefieren por otras características que al parecer compensan la susceptibilidad a esta enfermedad. 
En la Tabla 3 se aprecian las variedades que fueron sembradas durante la campaña 1997-98 en las tres provincias analizadas. La preferencia por variedades sembradas varía de acuerdo al lugar. En Cajamarca, la variedad Liberteña es preferida por su moderada resistencia al tizón y su buena calidad culinaria. En zonas con mayor vinculación al mercado de la costa como Contumazá y San Miguel las variedades más sembradas (Yungay y Canchan), aunque susceptibles al tizón tardío, son preferidas por su rendimiento, precocidad y aceptación en el mercado.

Tabla 3. Variedades de papa sembradas durante la campaña 1997-1998.

\begin{tabular}{|l|l|l|l|l|}
\hline $\begin{array}{l}\text { Campos } \\
\text { sembrados con: }\end{array}$ & Cajamarca & Contumazá & San Miguel & Promedio \\
\hline Amarilis & $13.2 \%$ & $43.8 \%$ & $37.0 \%$ & $29.0 \%$ \\
Canchan & $9.4 \%$ & $53.1 \%$ & $54.4 \%$ & $35.9 \%$ \\
Chaucha & $0.0 \%$ & $3.1 \%$ & $34.8 \%$ & $13.0 \%$ \\
Liberteña & $69.8 \%$ & $21.9 \%$ & $23.9 \%$ & $42.0 \%$ \\
Perricholi & $20.8 \%$ & $3.1 \%$ & $4.4 \%$ & $10.7 \%$ \\
Yungay & $28.3 \%$ & $65.6 \%$ & $41.3 \%$ & $42.0 \%$ \\
Otras & $34.0 \%$ & $21.9 \%$ & $34.8 \%$ & $32.3 \%$ \\
\hline
\end{tabular}

Variedades por campo: 1 variedad (15\%), 2-4 variedades (85\%). Los porcentajes indicados no suman $100 \%$ porque la mayoría de agricultores sembraban de 2 a 4 variedades en el mismo campo.

\section{El uso de fungicidas:}

De los 131 informantes, 95.4\% de ellos aplicó por lo menos en una oportunidad algún producto químico. En realidad, la mayoría aplicó fungicidas (94.7\%). Esto es similar a lo reportado por Crissman et al (1998) para la zona norte del Ecuador que es una zona papera comercial; pero diferente a lo mencionado por Ortiz et al (1996) que indica que en comunidades de subsistencia sólo $30 \%$ de agricultores tiene posibilidad de usar pesticidas. Debido a la variabilidad de condiciones agroclimáticas y socioeconómicas de los Andes no es posible hacer generalizaciones. 
De la muestra analizada se obtiene que los agricultores fumigaron entre 0 a 14 veces con un promedio de 6.6 veces durante la campaña. Sin embargo, cabe aclarar que la campaña 1997-1998 estuvo fuertemente afectada por el fenómeno climático de El Niño, lo que incrementó significativamente las lluvias en la zona. Esto ocasionó, según los agricultores, que tuvieran que aplicar el doble de lo normal. El número de aplicaciones, sin embargo, varía según la zona y la disponibilidad de recursos de los agricultores. En realidad, la mayoría de ellos (63\%) aplicó entre 1 a 6 veces, un 32\% aplicó entre 6 a 10 veces y sólo un 5\% aplicó entre 11 a 14 veces (estos últimos agricultores semi-comerciales ubicados en Contumazá y San Miguel).

La mayoría de aplicaciones se concentró entre diciembre a marzo. Se aprecia que la primera aplicación fue hecha aproximadamente entre un mes a un mes y medio después de la siembra, sin embargo hay algunos agricultores que aplicaron aun el mismo día de la siembra y otros aplicaron hasta más de dos meses después. En promedio los agricultores aplicaron fungicidas cada 7 días durante la campaña, lo cual es más frecuente de lo que ellos normalmente aplican en una campaña sin exceso de lluvia que es cada 12 a 14 días aproximadamente. La mayoría de agricultores que aplicaron entre 1 a 6 veces, lo hicieron entre el primer y tercer mes después de la siembra. Aparentemente, es durante esta etapa que el agricultor evalúa la respuesta de la variedad al tratamiento y decide continuar aplicando, según el clima y si es que tiene los recursos necesarios.

Respecto al tipo de productos utilizados en Cajamarca, se pudo apreciar que $51.3 \%$ de informantes usaron fungicidas de contacto, $1.4 \%$ usaron fungicidas sistémicos y $47.3 \%$ de ellos usaron productos que tienen la combinación de productos sistémicos y de contacto. La Tabla 4 muestra los diferentes ingredientes activos que se pudieron encontrar durante este estudio. Mancozeb fue el producto activo más usado (un total de $69.2 \%$ de aplicaciones), seguido del metalaxyl usado en $42.3 \%$ de aplicaciones y de propineb con $19.4 \%$ de aplicaciones.

Otro aspecto importante sobre el uso de fungicidas está relacionado a las dosis que utilizan los agricultores. La evidencia registrada indica que los agricultores utilizan en general menos de las dosis recomendadas por los fabricantes. La Figura 2 muestra que esto depende del producto que se utilice; pero, en el caso de productos que combinan metalaxyl con mancozeb que fueron los más usados, se aprecia que las dosis son ligeramente menores al 50\% de lo recomendado, generalmente debido a que estos productos son los 
más caros en el mercado. Las dosis de otros productos son mayores o menores al 50\% pero en general no llegan al $100 \%$ de lo recomendado. En general se apreció que la razón de usar menos dosis está relacionada a la disponibilidad de dinero para comprar los productos en la cantidad apropiada y oportuna.

Tabla 4. Ingredientes activos usados en la zona en estudio para el control del tizón tardío ( $\mathrm{N}=887$ aplicaciones).

\begin{tabular}{|l|l|c|}
\hline \multicolumn{1}{|c|}{ Tipo } & \multicolumn{1}{|c|}{ Ingrediente activo } & $\begin{array}{c}\text { Frecuencia (\% de } \\
\text { aplicaciones) }\end{array}$ \\
\hline Sistémico & benalaxil & 1.4 \\
\hline Contacto & mancozeb & 24.4 \\
& propineb & 19.4 \\
& metiram & 6.7 \\
& manganeb y sulfato de cobre & 0.8 \\
\hline Sistémico & metalaxyl + mancozeb & 42.2 \\
combinado con & dimethomorph + mancozeb & 2.0 \\
Contacto & fentín acetato + maneb & 1.4 \\
& cymoxanil + hidróxido de cobre & 1.0 \\
& fentín acetato + mancozeb & 0.3 \\
& cymoxanil + mancozeb & 0.2 \\
& cymoxanil + propineb & 0.2 \\
& & \\
\hline
\end{tabular}

Los datos colectados indican que en promedio los agricultores gastaron alrededor de 305 soles por hectárea (100 US\$) en la compra de fungicidas durante la campaña agrícola 1997-1998. Sin embargo, hubo gran variabilidad respecto a la inversión en fungicidas. Hubieron agricultores que no invirtieron en el control del tizón (5.3\%) hasta aquellos que invierten entre 200 a 500 US\$ por hectárea para controlar la rancha (12.9\%). Aproximadamente la mitad de los agricultores (51.1\%) gastaron entre 1 a 200 soles por hectárea (entre 1 a 66 US\$/ha) lo cual no es una inversión mayor comparada con otras zonas comerciales de los Andes.

\section{Factores que influyen en el uso de fungicidas:}

La Tabla 5 muestra los resultados de una regresión múltiple en la cual el uso de fungicidas fue considerada el factor dependiente y otras variables como factores independientes. Se deduce de este cálculo que la edad de los agricultores, el nivel de educación, el tamaño de la finca, la fecha de siembra y el lugar influyen significativamente en el uso de fungicidas. Por ejemplo, se puede decir que los agricultores más jóvenes tienden a aplicar más fungicidas, en la misma forma los agricultores con mayor nivel educativo y aquellos que tienen fincas más grandes. Los agricultores en Contumazá y San Miguel aplican 
más frecuentemente que los agricultores de Cajamarca, lo cual se debe a que las dos primeras provincias tienen condiciones climáticas más apropiadas para el desarrollo de la enfermedad, obligando a los agricultores a fumigar más frecuentemente. Además, en estas provincias hay más agricultores con vínculos comerciales y tienden a invertir más para el control del tizón tardío. Por otro lado, aquellos agricultores que siembran más temprano en la campaña agrícola aplican menos fungicidas, debido a que existe menos presencia de la enfermedad. Algo que es necesario resaltar es que, según los datos colectados, no existe evidencia que el uso de variedades resistentes tienda a disminuir el uso de fungicidas. Observaciones realizadas en el campo mostraron que los agricultores combinan variedades susceptibles y resistentes en forma contigua en el mismo campo y tienden a controlar el tizón de manera uniforme ya que el costo adicional de fumigar pequeñas parcelas es relativamente bajo. También hay un efecto psicológico que les da tranquilidad cuando fumigan, así se trate de variedades resistentes.

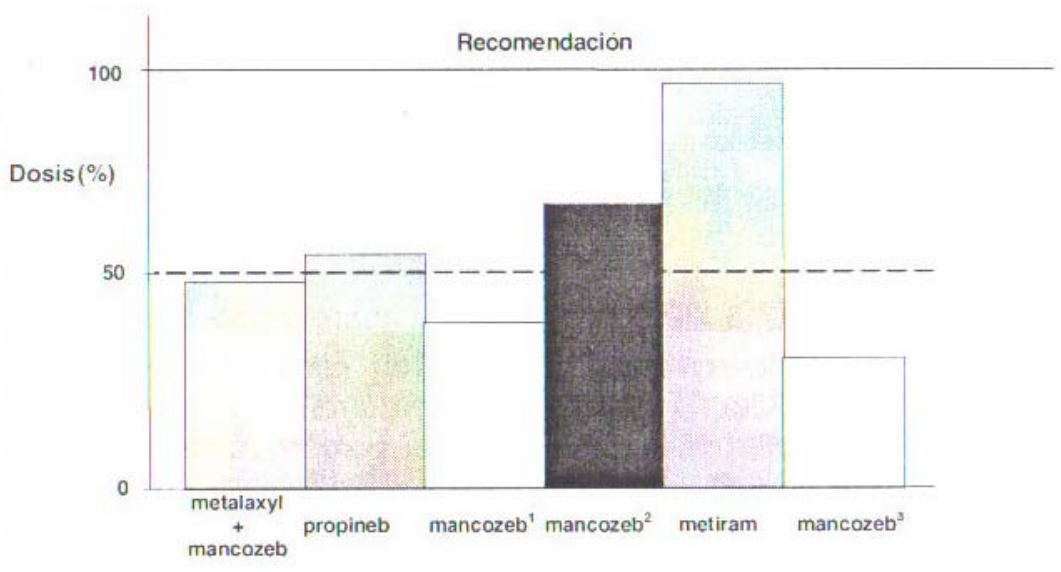

Figura 2. Dosis de los ingredientes activos más usados en la zona. (En el caso de mancozeb, los números indican diferentes productos comerciales.) 
Tabla 5. Factores que influyen en el uso de fungicidas.

\begin{tabular}{|c|c|c|}
\hline Variable & Coeficiente & Significancia \\
\hline \begin{tabular}{|l|} 
Edad \\
Educación \\
Mano de obra familiar \\
Área de la finca \\
Variedades:
\end{tabular} & $\begin{array}{l}-0.04 \\
0.26 \\
0.01 \\
0.53\end{array}$ & $\begin{array}{l}-1.95^{\star} \\
0.33^{\star \star} \\
0.06 \\
2.16^{\star \star}\end{array}$ \\
\hline Amarilis & -3.66 & -0.66 \\
\hline Canchán & 0.47 & 0.84 \\
\hline Chaucha & 1.38 & 1.63 \\
\hline Liberteña & -0.35 & -0.62 \\
\hline $\begin{array}{l}\text { Perricholi } \\
\text { Yungay }\end{array}$ & 0.93 & $\begin{array}{l}1.22 \\
-0.37\end{array}$ \\
\hline Fecha de siembra & 0.21 & $3.27^{* \star *}$ \\
\hline Lugares. Contumazá & 3.34 & 4.24 *** \\
\hline San Miguel & 3.38 & $4.49 * * *$ \\
\hline Constante (Caiamarca) & -286.03 & $-3.22 * \star \star$ \\
\hline Número de observaciones & & 131.00 \\
\hline$R^{2}$ & & 0.56 \\
\hline
\end{tabular}

Nota: La variedad de referencia es el grupo de variedades nativas. El lugar de referencia es la provincia de Cajamarca.

\section{La relación entre el daño del tizón tardío y el rendimiento de la papa}

\section{La severidad del daño por tizón:}

Durante la campaña agrícola se evaluaron campos de papa para determinar la severidad de ataque del tizón tardío. La evaluación se basó en la estimación visual periódica del porcentaje de área verde afectada por la enfermedad. Se trató de tener por lo menos tres evaluaciones por parcela durante la campaña, sin embargo, las condiciones climáticas adversas impidieron que esto se concretara y en la mayoría de casos se evaluó el daño sólo en dos oportunidades.

Considerando 188 evaluaciones que se realizaron en total, se puede graficar la severidad respecto a la fecha de siembra (Figura 3), se aprecia que la tendencia de incremento de severidad en el tiempo tiende a ser una curva (promedio de severidad). Teóricamente, esta curva debería ser más pronunciada, sin embargo, las diferentes fechas de siembra, diferentes lugares, el uso de diferentes variedades $y$ de diferentes fungicidas influyen para tener una curva no tan pronunciada. Posiblemente una tercera evaluación hubiera permitido observar una evolución más pronunciada de la enfermedad. 


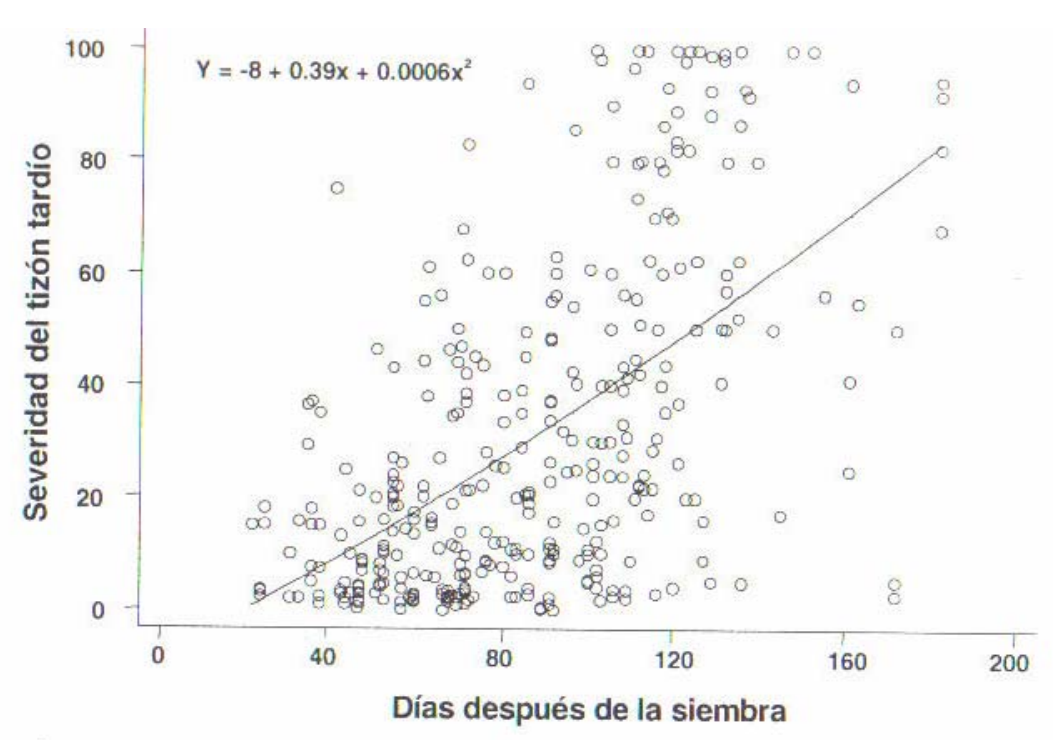

Figura 3. Evolución de la severidad del daño respecto a la fecha de siembra en las parcelas evaluadas ( $N=188$ evaluaciones).

Cuando se toma la curva de severidad promedio y se mide las desviaciones según cada variedad (Figura 4), se aprecia que hay variedades que tienen una desviación promedio negativa, es decir tuvieron menos daño. Este es el caso de la variedad Amarilis que muestra una desviación negativa significativa (lo cual muestra su resistencia), seguido de la variedad Liberteña. Las variedades Perricholi y Chaucha muestran desviaciones ligeramente negativas, Io cual sugiere también ciertos niveles de resistencia a nivel de campo. Por otro lado hay variedades que muestran desviaciones promedios positivas, es decir que tuvieron más daño, tal es el caso de Canchan, Yungay y otras variedades nativas. En resumen, se puede decir que hay evidencia que demuestra diferencias significativas de severidad a nivel de campo entre las variedades utilizadas por los agricultores, lo cual sugiere que la resistencia genética puede jugar un papel importante en la lucha contra el tizón tardío. 


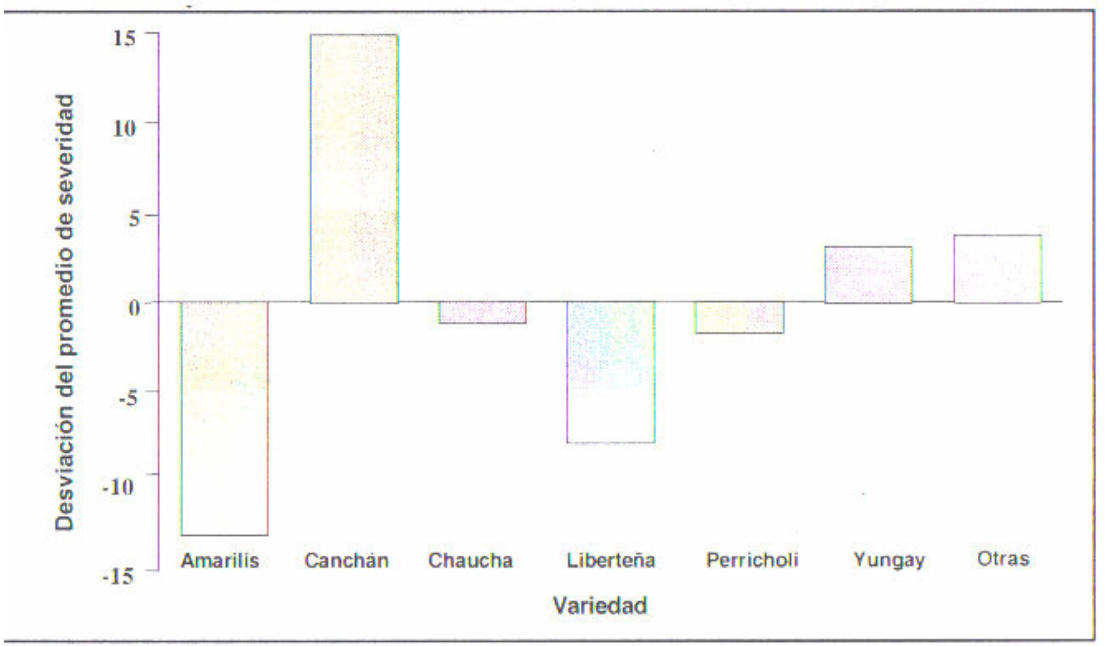

Figura 4 Desviación del promedio de severidad según la variedad.

\section{Los factores que influyen en la severidad del daño:}

Se realizó una regresión múltiple para determinar los factores que influyen en la severidad de daño por tizón (Tabla 6). Los factores considerados fueron la fecha de siembra, fecha de evaluación del daño en días después de la siembra, el número de aplicaciones de fungicidas, las variedades y los lugares (provincias). La fecha de siembra estuvo positivamente correlacionada con la severidad, es decir que sembrando un mes después (por ejemplo en diciembre en lugar de noviembre), la severidad del daño se incrementaría en 15\%. Esto explica el por qué algunos agricultores prefieren sembrar entre junio a agosto y tienen menos problemas con tizón. La fecha de evaluación del daño en días después de la siembra también estuvo correlacionada significativamente con dicho daño, ya que la enfermedad tiende a incrementarse con el tiempo. El número de aplicaciones de fungicida está correlacionada negativa y significativamente con el daño. Es decir a mayor número de aplicaciones menor daño. Luego se tiene que hay variedades que fueron menos atacadas por la enfermedad; es decir la selección de la variedad usada estuvo significativamente correlacionada con el daño (controlando por otros factores). Este fue el caso de las variedades Amarilis, Liberteña y Chaucha (correlación negativa). Por otro lado están las variedades que muestran una correlación positiva significativa, es decir tienden a tener mayor daño. Este es el caso de Canchan y Yungay. Perricholi no está asociada significativamente al 
daño. Finalmente, el lugar (provincia) también está correlacionado al daño existiendo mayor daño en San Miguel,y Contumazá que en Cajamarca.

Tabla 6. Factores que influyen en la severidad del daño por tizón.

\begin{tabular}{|l|c|c|}
\hline Variable & Coeficiente & Significancia \\
\hline Días después de la siembra & 0.31 & $2.06^{\star \star}$ \\
Días después de la siembra (cuadrado) & 0.002 & $3.10^{\star \star \star}$ \\
Fecha de siembra & 0.55 & $10.18^{\star \star \star}$ \\
Aplicación de fungicidas & -1.21 & $-2.88^{\star}$ \\
Variedades: & & \\
Amarilis & -14.40 & $-3.74^{\star}$ \\
Canchan & 15.20 & $4.10^{\star}$ \\
Chaucha & -9.45 & $-1.84^{\star}$ \\
Liberteña & -8.86 & $-2.70^{\star \star \star}$ \\
Yerricholi & 1.01 & 0.18 \\
Yungáy & 4.35 & 1.18 \\
Cugares: & 10.08 & $2.45^{\star \star}$ \\
Santumazá & & \\
& 8.58 & $2.59^{\star \star \star}$ \\
\hline Constante (Cajamarca) & -7568.41 & -10.18 \\
\hline Número de observaciones & & 355.00 \\
$\mathbf{R}^{2}$ & & 0.54 \\
\hline
\end{tabular}

Los datos presentados anteriormente sugieren que los agricultores tienen tres estrategias principales para reducir el daño del tizón. La primera es tratar de sembrar durante la época seca, lo cual está limitado a campos con humedad suficiente. La segunda es el uso de fungicidas. Por ultimo, el uso de variedades resistentes.

\section{La relación entre severidad de daño y rendimiento:}

El rendimiento promedio evaluado en 122 parcelas fue de 4.9 t/ha, lo cual es menor que el rendimiento promedio para la zona de Cajamarca que oscila entre 7 a 10 t/ha según PIDAE (1996). El factor que influyó en este bajo rendimiento fue el exceso de lluvias lo que agudizó el ataque del tizón tardío. Esto ocasionó que aproximadamente $17 \%$ de parcelas tuvieran rendimientos menores a $1 \mathrm{t} / \mathrm{ha}$, lo cual se puede considerar como pérdida total del cultivo. En $25 \%$ de parcelas, los rendimientos fueron 1 a 3 t/ha. $27 \%$ de parcelas tuvieron un rendimiento entre 3 a 6 t/ha. 13\% de parcelas rindieron entre 6 a 9 t/ha y $17 \%$ tuvo rendimientos entre 10 a 22 t/ha. 
El rendimiento puede ser influido por una serie de factores como clima, fertilidad, variedad, ataque de plagas y enfermedades, manejo, época se siembra, etc. Sin embargo, a pesar de la gran variabilidad de casos evaluados, se pudo observar una clara relación entre el daño causado por tizón y el rendimiento por hectárea de la papa.

Si se establece una curva para expresar la relación entre rendimiento por hectárea y el daño promedio obtenido (Figura 5) se aprecia que daño y rendimiento están relacionados. Un 20\% de incremento de severidad tiende a reducir 1 t/ha de rendimiento. Es decir que en promedio una variedad con una severidad de $20 \%$ rendiría dos toneladas más que esa u otra variedad con daño promedio de $60 \%$, lo cual significaría un incremento de ingreso de aproximadamente 240 dólares por hectárea considerando un precio promedio por tonelada de papa de 140 US\$. Sin embargo, esto depende mucho de la variedad que se utilice. Por ejemplo, la variedad Amarilis promedia aproximadamente $14 \mathrm{t} / \mathrm{ha}$ de rendimiento comparada con la variedad Canchan que promedia 2 t/ha o con Liberteña que promedia 5 t/ha, Perricholi con 4 t/ha o Yungay con 3.5 t/ha. Esto demuestra la potencialidad que existe de obtener incrementos de rendimiento cuando se utilizan variedades resistentes al tizón tardío bajo condiciones similares de manejo del cultivo y de presión de la enfermedad.

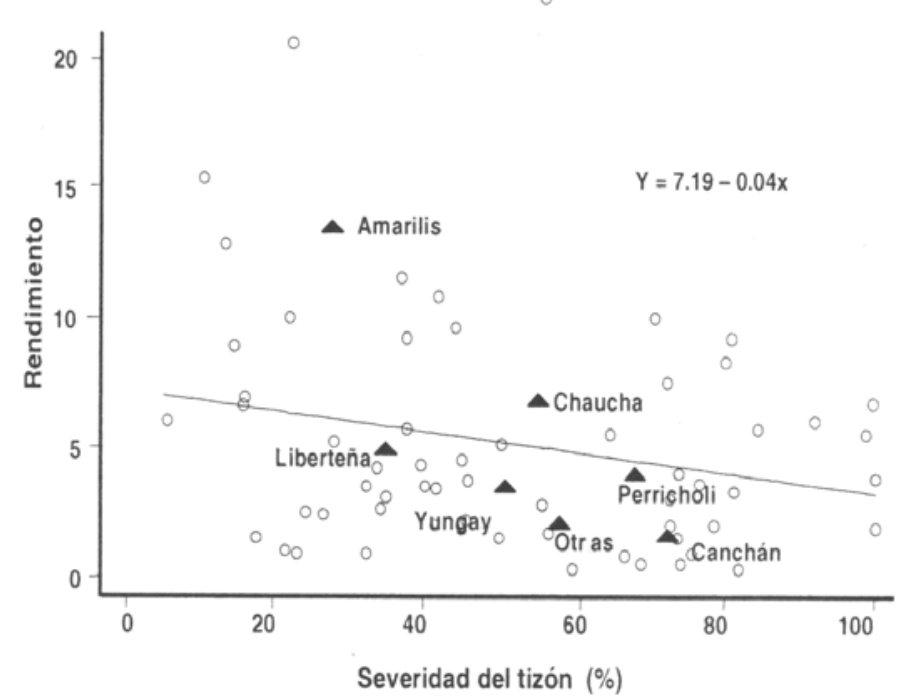

Figura 5. Relación entre rendimiento y severidad promedio en las parcelas evaluadas. 


\section{Conclusiones}

La evidencia presentada en las anteriores secciones permite obtener las siguientes conclusiones:

1. El tizón tardío es el problema más importante del cultivo de papa que enfrentan los agricultores en la zona de Cajamarca, Contumazá y San Miguel. El daño causado por el tizón está correlacionado positiva y significativamente con la fecha de siembra y la fecha de evaluación. Esto significa que el riesgo de ataque se incrementa si los agricultores siembran más tarde en la campaña agrícola. El ataque se incrementa entre enero y marzo. También existe correlación positiva y significativa entre la severidad de daño y el uso de variedades susceptibles y entre severidad y los lugares (en San Miguel y Contumazá hubo más daño). Por el contrario, la aplicación de fungicidas y el uso de variedades resistentes esta correlacionado significativa pero negativamente con el daño. Es decir, a mayor número de aplicaciones menor daño y hay variedades que tienen menor daño en promedio en los lugares evaluados. Esto sugiere que un programa de manejo integrado del tizón tardío debería incluir como componentes esenciales la resistencia varietal y el uso apropiado de fungicidas.

2. No obstante que el tizón tardío es el principal problema en el cultivo de la papa desde la perspectiva de los agricultores, ellos también priorizan otras plagas que tienen importancia según la zona. Tal es el caso del gorgojo de los Andes (Premmnotrypes spp), la pulga saltona (Epitrix spp), la polilla de la papa (Simestrishema tangolias y Phthorimaea operculella), otros insectos de hoja y suelo, otros hongos (Alternaria y Phoma) y pudrición de tubérculos. Esta diversidad de plagas sugiere que un programa de manejo integrado debería tener la suficiente flexibilidad para ser adaptado a las condiciones y a las plagas locales. Por otro lado, la papa es uno entre varios cultivos y crianzas que ocupan el tiempo de los agricultores quienes, además, trabajan fuera de la finca o en artesanía y comercio. La diversidad de actividades de los agricultores plantea la necesidad de que los programas de manejo integrado de plagas tengan en cuenta los objetivos de la familia campesina y la mano de obra disponible como factores que podrían influir en su adopción.

3. Los agricultores utilizan su propio conocimiento y tecnología para controlar al tizón tardío de la papa. Conocimiento basado en su 
experiencia y en los contactos con organizaciones de extensión. Sin embargo, ellos carecen de información importante referida al origen de la enfermedad, la identificación correcta de síntomas y el uso apropiado de fungicidas según la variedad. Un programa de manejo integrado del tizón debería estar orientado a mejorar lo que el agricultor ya conoce y practica correctamente. Además de facilitar que ellos puedan tener acceso a la información y el conocimiento que les hace falta.

4. El uso de fungicidas es la práctica más común para el control del tizón; sin embargo, las dosis que utilizan son generalmente menores a las recomendadas, lo cual posiblemente influye en la eficiencia de control. La forma de aplicación y la combinación apropiada de fungicidas de contacto y sistémicos es algo desconocido y por lo tanto no practicado por la mayoría de agricultores. El costo de usar fungicidas no es extremadamente alto, por lo tanto, no se debería esperar una significativa reducción del costo de control como una potencial fuente de beneficio. Por el contrario, el enfoque debería estar orientado a incrementar el rendimiento a través del uso de variedades resistentes combinadas con el uso apropiado de fungicidas y otros posibles métodos de prevención y control.

5. La evidencia presentada sugiere que hay una serie de hipótesis que requieren ser probadas. Primero, saber si mejorando el conocimiento de los agricultores sobre las plagas y su manejo se lograría que ellos mejoren sus decisiones para el control; la segunda se refiere a que si introduciendo variedades resistentes se reduciría significativamente el uso de fungicidas; la tercera se refiere a saber si se podrían desarrollar y difundir métodos alternativos de control como el manejo integrado (del tizón y otras plagas de la papa) que fueran más rentables para los agricultores.

\section{Referencias Bibliografías}

1. Bentley, J. 1990. Conocimiento y experimentos espontáneos de campesinos hondurenos sobre el maíz muerto. En: Manejo Integrado de Plagas. Costa Rica. Vol.17. p. 16-26.

2. Crissman, C.; Espinosa, P.; Ducrot, C.; Cole, D.; Carpio, F. 1998. The case study site: Physical, health, and potato farming systems in Carchi province. En: Economic, environmental, and health trade offs in agriculture: Pesticides and the sustainability of Andean Potato Production. C. Crissman, J. Antle y S. Capallo (eds.). Kluwer Academic Publisers - CIP. p. 86-119. 
3. Gálvez, D.; Gallardo, L.; Rodríguez, J. 1988. Calendario Agrícola en el área del proyecto piloto de ecosistemas andinos. A. Koeler (ed.). Proyecto Piloto de Ecosistemas Andinos. Cajamarca, Perú

4. Horton, D.; Tardieu, F.; Benavides, M.; Tomassini, L.; Accatino, P. 1980. Tecnología de la producción de papa en el valle de Mantaro, Perú. Departamento de Ciencias Sociales. Documento de Trabajo 1980-1 Centro Internacional de la Papa . Lima, Perú.

5. Instituto Nacional de Estadística e Informática (INEI). 1996. III Censo Nacional Agropecuario. Resultados definitivos. Dirección técnica de Censos y Encuestas. INEI-Ministerio de Agricultura. Lima, Perú.

6. IPM-Task Force. 1992. Integrated pest management in developing countries. Experience and prospects. Natural Resources Institute. UK.

7. Krueger, R. 1994. Focus groups. A practical guide for applied research. SAGE Publications. USA.

8. Morgan, D.; Krueger, R. 1993. When to use focus groups and why. En: Successful focus groups. Advancing the state of the art. D. Morgan (ed.). Sage Publications. USA. p. 3-19.

9. Nelson, R.; Fredrix, M.; Thiele, G.; Mund, C. 1998. Improving farmers' disease management decision through participatory research and training. En: Invited Papers. Abstraéis Volume $1.7^{\text {th }}$ International Congress of Plant Pathology. UK. p 3.1.4s.

10. Ortiz, O.; Valdez, A. 1993. Enfoque de sistemas y metodología participativa para desarrollar medios escritos de comunicación agrícola. El caso de la papa en la agricultura de subsistencia. Revista Latinoamericana de la Papa. Vol. 5/6. p. 103-121.

11. Ortiz, O.; Alcázar, J.; Catalán, W.; Villano, W.; Cerna, V.; Fano, H.; Walker, T. 1996. Economic impact of IPM practices on the Andean potato weevil in Perú. En: Case studies of the economic impact of CIP-related technology. T. Walker and C. Crissman (eds.). International Potato Center. Lima, Perú. p. 95-110

12. Ortiz, O. 1997. The information system for IPM in subsistence 
potato production in Perú: experience of introducing innovative information in Cajamarca Province. Ph. D. Thesis. ARDD. The University of Reading. Reino Unido. 367 p.

13. PIDAE, 1995. La Encañada: caminos hacia la sostenibilidad. Centro Internacional de la papa. Lima, Perú.

14. Röling, N.; Van de Fliert, E. 1994. Transforming extension for sustainable agriculture. The case of integrated pest management in rice in Indonesia. Agriculture and Human Values. Vol. 11. No 2-3. p. 96-108.

15. Sánchez, R.; Equipo tinta. 1993. Ecología, producción y desarrollo campesino. Taller de Investigación en Tecnologías Agroecológicas. Lima, Perú.

16. Torres, J.L. 1896. Cartilla de agricultura del Perú. Imprenta El Comercio. Lima, Perú.

17. Van de Fliert, E. 1993. Integrated Pest Management: farmer field schools generate sustainable practices. A case study in Central Java evaluating IPM training. Agricultural University Wageningen. The Netherlands. 